\title{
An Indoor Bluetooth-based Positioning System using Region Division and Fingerprinting
}

\author{
Siquan $\mathrm{Hu}^{1,2, a}$, Wenhui Lei $\mathrm{e}^{1, \mathrm{~b}}$ and Chundong She $\mathrm{S}^{3, \mathrm{c}}$ \\ ${ }^{1}$ School of Computer and Communication Engineering, University of Science and Technology \\ Beijing, Beijing, 100083, China \\ ${ }^{2}$ Chengdu Visual Microcosm Technology Ltd. Chengdu, 610072, Sichuan, China \\ ${ }^{3}$ School of Electronic Engineering, Beijing University of Posts and Telecommunications, Beijing, \\ 100876, China \\ ahusiquan@ustb.edu.cn, ${ }^{b}$ leiwenhui@outlook.com, ${ }^{\mathrm{c}}$ shurcd@vip.sina.com
}

Keywords: real-time indoor location; region division; RSSI; BLE.

Abstract. Indoor location has become an active research area and developing direction in the last decade, and BLE method is commonly used in indoor positioning. This paper mainly presents an indoor location system based on Bluetooth by using the method of region division and fingerprinting. The experimental results demonstrate that in the typical office scene, the proposed localization algorithm outperforms the single mode localization with accuracy and response.

\section{Introduction}

With the development of communication technology, Location Based Service (LBS) is more and more widespread. Location systems are one of the most promising fields in mobile computing which allow the new location-aware mobile applications are developed. Furthermore, automatic handover procedures based on the user location are necessary to provide quality of service in wireless networks especially in cases of traffic overloads [1]. The GPS can bring a satisfactory positioning result in outdoor environment while the GPS is not suitable for indoor positioning. There are many wireless positioning technologies currently used in indoor scene, such as infrared, Bluetooth, wireless sensor network and WLAN. Bluetooth is a more pervasive technology among mobile phones and PDAs [2]. A major advantage of using Bluetooth is that it is a technology with high penetration in society. Devices such as cellphones, tablets and computers normally come equipped with the technology as standard [3].

Wireless localization algorithms can be roughly divided into two categories, Range-based and Range-free localization algorithms [4]. The precision of signal transmission mainly depends on the channel attenuation model and whether the model corresponds with the current complex layout and structure. However, there are many factors that can affect transmission model, such as distance, signal attenuation, multipath effect, etc. Fingerprinting is a common technique for indoor positioning using short range Radio Frequency (RF) technologies such as Wireless Location Area Network (WLAN) and Bluetooth (BT) [5]. Location fingerprinting method is to match the signal characteristic value from terminal measurement with the previous sample records in the database, and location information is obtained by the matching algorithm. We should point out that the accuracy of location fingerprinting positioning is higher than the signal transmission model. However, using location fingerprinting method needs a great amount of calculation, which will be a challenge to the indoor location services. With the rise of BLE technology, the BLE node's miniaturization, low cost, easy deployment can be used to indoor location. Therefore, this paper proposes an indoor location system based on BLE combined with fingerprinting method and region division.

\section{Related Technologies and Methods}

BLE and RSS Ranging Model. Bluetooth wireless technology was created by the company Ericsson in 1994. The main use of Bluetooth technology is to exchange data over short distances using radio 
transmissions. The latest version of Bluetooth is Bluetooth 4, which was released in 2013 and is under revision since 2014, aiming at higher transfer speeds and less power consumption. BLE is especially designed to ensure low energy consumption and inexpensive devices, as the classic Bluetooth cannot achieve the low power requirements that needed for devices powered only by button-cell batteries. The architecture is very similar to the classic Bluetooth architecture and is split into three parts: controller, host and application.

The wireless signal of the signal strength increases with the increase of propagation distance attenuation, the closer the receiver to the sender, the stronger the signal strength will be; The further the receiver from the sender, the weaker the signal strength will be. According to the signal strength and wireless signal fading model, we can estimate the distance from the sender to the receiver. According to the multiple estimated distances, the position of the terminal can be calculated.

To calculate the distance based on the RSSI values, we can use a simple loss model shown in Fig. 1 and Eq.1. The positioning model requires, in addition to the two RSSI model parameter, A and $\mathrm{n}$ which are discussed in this section.

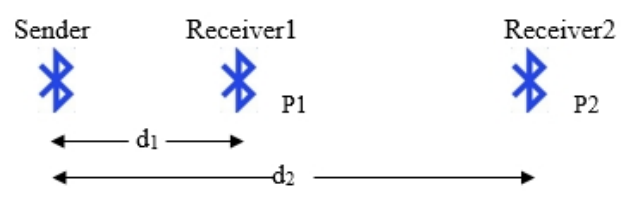

Fig.1 Free space path loss model

$$
P_{2}=P_{1}+10 n\left(\log _{10} d_{1}-\log _{10} d_{2}\right)
$$

After operation, we can get the Eq.2.

$$
R S S I=-\left(A+10 n \times \log _{10} d\right)
$$

In the case of stationary or low speed receiving, a simple average filter is used to improve the measurement accuracy as Eq.3. However, In the case of high speed receiving, Kalman filtering will be used.

$$
\overline{R S S I}=\frac{1}{n} \sum_{i=0}^{i=n} R S S I_{i}
$$

Region Division. The radio coverage of a BLE node is defined as a region, the size of the area can be modified by adjusting BLE node transmission power. Fig. 2 shows the division of region by using BLE nodes. In Fig.2, BLE1, BLE2, and BLE3 have different transmit power, corresponding region 1, region 2 and region 3. Every BLE node broadcast its own regional information periodically. The target is the terminal contains the BLE chip. If the target is within a region, it will receive the region information broadcasted by the BLE node.

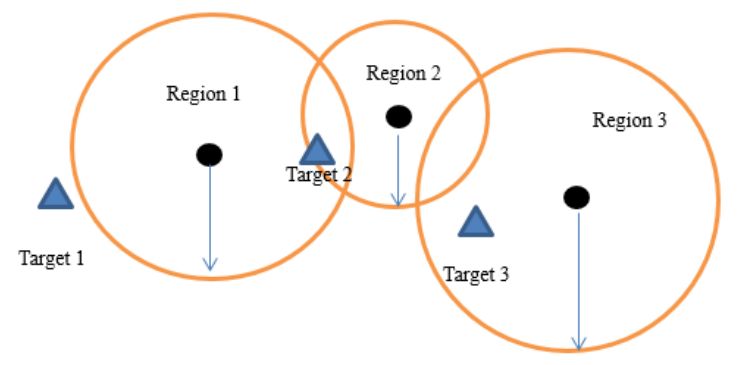

Fig.2 The region division 
The principle of region division positioning is shown in Fig.2. If the target is beyond the region, as target 1, the positioning server will estimate the location of target from all the sampling record. However, actually, maximum range of a node is 10 meters, being enough to make the house not uncovered area. If target is within the region, like target 2 or target 3 , the server will match the position from the corresponding sampling. It can avoid the server every time estimate target position from the sampling record, reduce the computational complexity of the server.

\section{System Architecture}

The system adopts $\mathrm{C} / \mathrm{S}$ structure and positions by using fingerprint. The system is composed of wireless AP, BLE nodes, mobile terminal, and positioning server. The WLAN is used as the communication between mobile terminals and positioning server and UDP (User Datagram Protocol) is considered to avoid the frequently switching wireless AP. BLE nodes are used for region division. Mobile terminal sends the list of signal strength and region information of BLE nodes to positioning server, positioning server calculates the location and returns it to mobile terminal.

\section{Information Processing and Positioning Estimation}

Fig.3 shows the information processing on the positioning server. When the server receives the request from the client, if the request contains the region information, the server matches the signal strength list to the fingerprints from the corresponding region, or matches it to the all fingerprints in database. Finally, the server returns the result to the terminal.

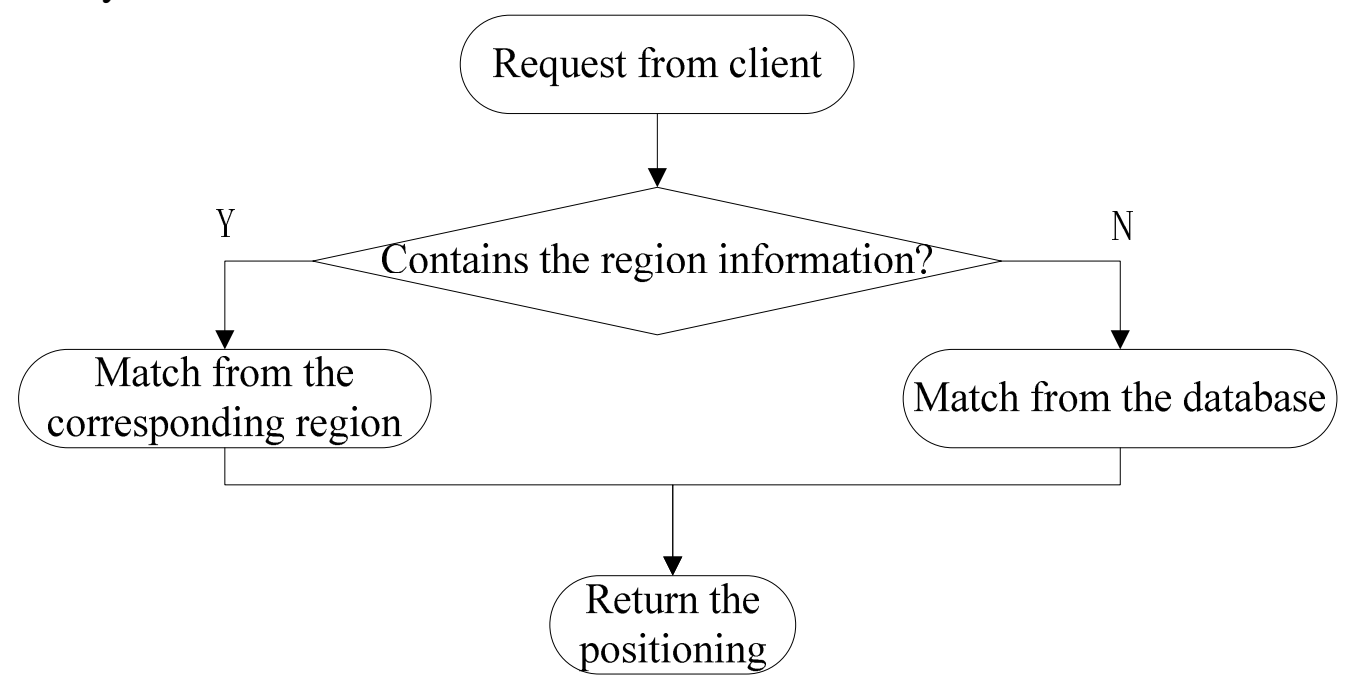

Fig.3 Data Processing

The algorithm in server is the KNN (the k-Nearest Neighbors algorithm), using Euclidean metric to represent the deviation between the request information and actual RSS, shown in Eq.4.

$$
d_{i}=\sqrt{\sum_{j=1}^{m}\left(R S S_{j i}-R S S_{j}^{\prime}\right)^{2}} \quad j=1,2, \ldots, m
$$

The RSSji is the jth signal strength at the ith position, the RSS $\mathrm{j}$ is the $\mathrm{jth}$ RSS', $m$ means the amount of APs. And finally, the server can estimate the position of client from the closest K sample as it shows in Eq.6: 


$$
(x, y)=\frac{\sum_{k=1}^{K} \frac{1}{d_{i}}(x, y)_{i}}{\sum_{k=1}^{K} \frac{1}{d_{i}}}
$$

\section{Experiment and Consequence}

To examine the accuracy of the system, we have setup the experimental environment in an indoor office open area showed inFig.4. We have set up 4 BLE anchor nodes to make sure that the nodes can cover the whole area. Fig.5 shows the training and test results. The results show that the proposed methods are valid in position estimation.

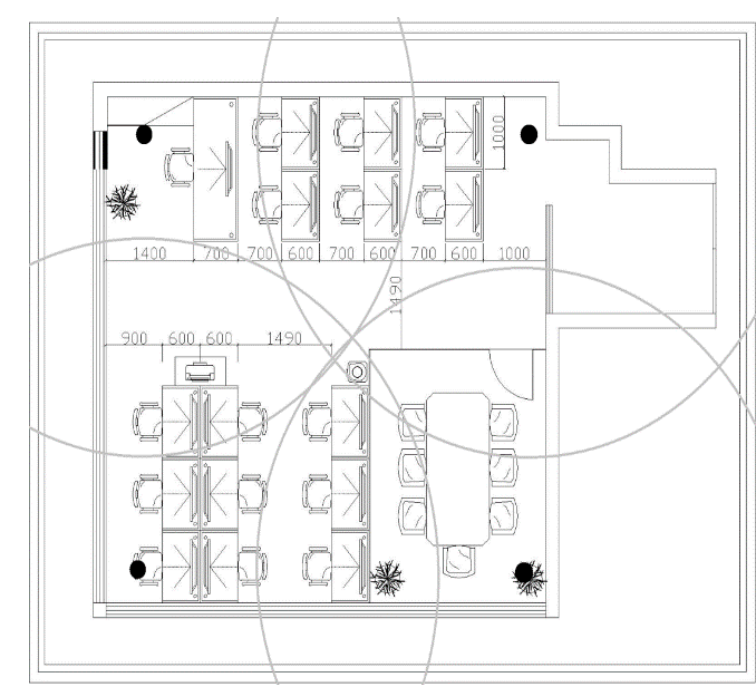

Fig.4 Experiment Environment

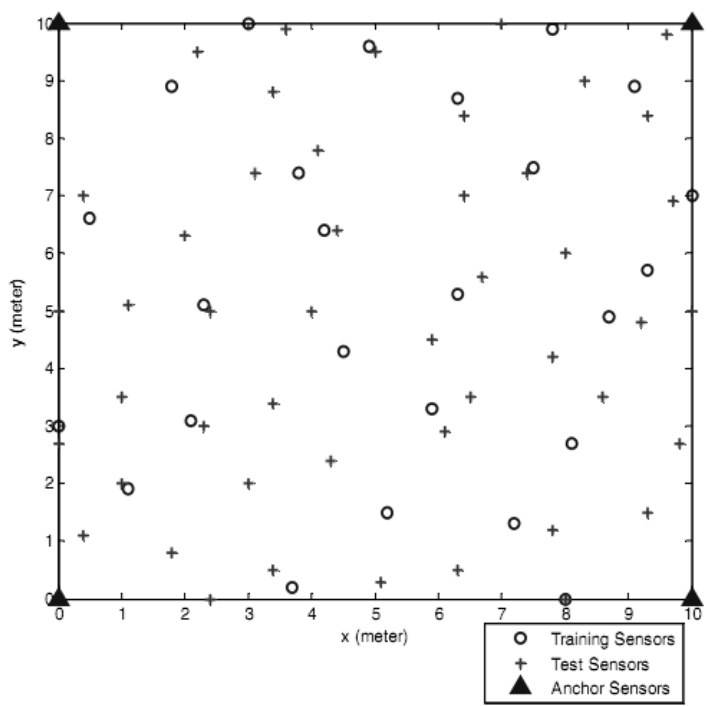

Fig.5 Training and Test

\section{Acknowledgements}

The study was supported by the National Natural Science Foundation of China (No. 91438120) and the Sichuan Science and Technology Support Program (No.2016GZ0339).

\section{References}

[1] S. Feldmann, K. Kyamakya, A. Zapater: Int. Conf. on Wireless Networks (2003), p.109

[2] M. Muñoz-Organero, P.J. Muñoz-Merino, C. D. Kloos: Mobile Info. Systems Vol. 8(2012), p.73

[3] E. Dahlgren, H. Mahmood: Evaluation of indoor positioning based on BluetoothR Smart technology, Master Thesis, Chalmers University of Technology, Sweden (2014)

[4] X. Zhu, Y. Feng: Communications and Network Vol. 5 (2013), p.37

[5] L. Pei, R. Chen, J. Liu, H. Kuusniemi, T. Tenhunen, Y. Chen: Journal of Global Positioning Systems Vol. 9 (2010), p.122 